\title{
Cavity-enhanced two-photon interference using remote quantum dot sources
}

\author{
V. Giesz, ${ }^{1}$ S. L. Portalupi, ${ }^{1, *}$ T. Grange,${ }^{2,3}$ C. Antón, ${ }^{1,4}$ L. De Santis,${ }^{1,5}$ J. Demory, ${ }^{1}$ N. Somaschi, ${ }^{1}$ I. Sagnes,,${ }^{1}$ A. Lemaître, ${ }^{1}$ \\ L. Lanco, ${ }^{1,6}$ A. Auffèves, ${ }^{2,3}$ and P. Senellart ${ }^{1,7, \dagger}$ \\ ${ }^{1}$ Laboratoire de Photonique et de Nanostructures, CNRS, UPR20, Route de Nozay, F-91460 Marcoussis, France \\ ${ }^{2}$ Université Grenoble Alpes, F-38000 Grenoble, France \\ ${ }^{3}$ CNRS, Institut Néel, "Nanophysique et Semiconducteurs" Group, F-38000 Grenoble, France \\ ${ }^{4}$ Departamento de Física de Materiales, Universidad Autónoma de Madrid, E-28049 Madrid, Spain \\ ${ }^{5}$ Université Paris-Sud, Université Paris-Saclay, F-91405 Orsay, France \\ ${ }^{6}$ Université Paris Diderot-Paris 7, F-75205 Paris Cedex 13, France \\ ${ }^{7}$ Physics Deparment, Ecole Polytechnique, Université Paris-Saclay, F-91128 Palaiseau Cedex, France \\ (Received 21 May 2015; revised manuscript received 3 September 2015; published 12 October 2015)
}

\begin{abstract}
Quantum dots in cavities have been shown to be very bright sources of indistinguishable single photons. Yet the quantum interference between two such bright quantum dot sources, a critical step for photon-based quantum computation, still needs to be investigated. Here, we report on such a measurement, taking advantage of a deterministic fabrication of the devices. We show that cavity quantum electrodynamics can efficiently improve the quantum interference between remote quantum dot sources: Poorly indistinguishable photons can still interfere with good contrast with high quality photons emitted by a source in the strong Purcell regime. Our measurements and calculations show that cavity quantum electrodynamics is a powerful tool for interconnecting several quantum dot devices.
\end{abstract}

PACS number(s): 78.67.Hc, 42.50.Ar, 42.50.Dv, 42.50.Pq

Recent years have seen impressive progress in the implementation of quantum functionalities using semiconductor quantum dots (QDs). These artificial atoms [1] have been used to generate flying quantum bits in the form of single photons [2] or entangled photon pairs [3,4], to map and optically manipulate the quantum information encoded onto a single stationary electron [5,6] or hole spin [7], as well as to implement optical logic gates [8-10]. The potential of QD-based single photon sources lies in their deterministic and pure quantum statistics, as opposed to the parametric down-conversion sources currently used in quantum optics. At saturation, the QD emits a single photon with a probability close to one and a vanishing probability of emitting a second photon. However, collecting single photons emitted by a QD is challenging: The collection efficiency is limited to $1 \%-2 \%$ for a QD in bulk [11] and to 5\%-10\% for a QD in a planar cavity structure. New strategies have been developed to reach high collection efficiencies (in the 40\%-90\% range) such as inserting the QD in one-dimensional photonic crystal or nanowire waveguides [11-14], in photonic crystal nanocavities [15], microdisk cavities [16], or micropillar cavities [17].

Since the first demonstration in 2002 [18], the coalescence of photons successively emitted by a single QD has been widely investigated. Various strategies have been developed to minimize the environment-induced dephasing (phonons, charge noise). One approach consists of using a strictly resonant excitation to create the carriers directly into the QD state and reduce the time jitter of the photon emission [9,19-23]. Another approach consists of using the Purcell effect by inserting the QD in a microcavity. The acceleration of spontaneous emission reduces the effect of dephasing

\footnotetext{
*Present address: Institut fur Halbleriteroptik und Funktionelle Grenzflachen, Universitat Stuttgart, Germany.

†pascale.senellart@1pn.cnrs.fr
}

$[17,18,23]$ and leads to an efficient extraction of photons: Ultrabright sources of highly indistinguishable photons were recently reported using this approach [17].

The scalability of a quantum network based on QDs relies on the possibility of interconnecting two QD devices. Pioneering steps have been made in this direction by investigating two-photon interference between single photons emitted by remote QDs in planar structures [24-27]. Under nonresonant excitation, the coalescence probability is limited to $25 \%-40 \%$ by the dephasing processes on each source [24-26]. The use of a resonant, spectrally narrow excitation line has led to a higher coalescence probability (up to 80\%) [27]. Such a technique has yet to be combined with an efficient extraction of the photons. Besides, such a spectrally narrow excitation results in an effective filtering of the events where both QD transitions are precisely at the laser frequency and is not compatible with a high brightness of the devices. In the present Rapid Communication, we demonstrate that the Purcell effect, which allows an efficient collection of photons, is also a powerful tool to improve the coalescence probability from remote sources. We study the quantum interference of remote QD bright sources, each of them consisting of a single InGaAs QD embedded in a microcavity. Accelerating the spontaneous emission of one source is shown to improve the two-source coalescence probability by efficiently overcoming the effect of pure dephasing of the other source.

Although it is an essential tool for scalable solid-state quantum information processing, remote QD source interference has been scarcely studied [24-27] and still needs to be studied for QD-cavity devices where a strong Purcell effect allows for the fabrication of the brightest sources of indistinguishable single photons to date [17]. Indeed, this requires control of both the spatial and spectral tuning of each QD to a given cavity mode as well as respective spectral tuning of the two devices. This highly challenging step is achieved here owing to a deterministic fabrication of the QD-cavity devices. 

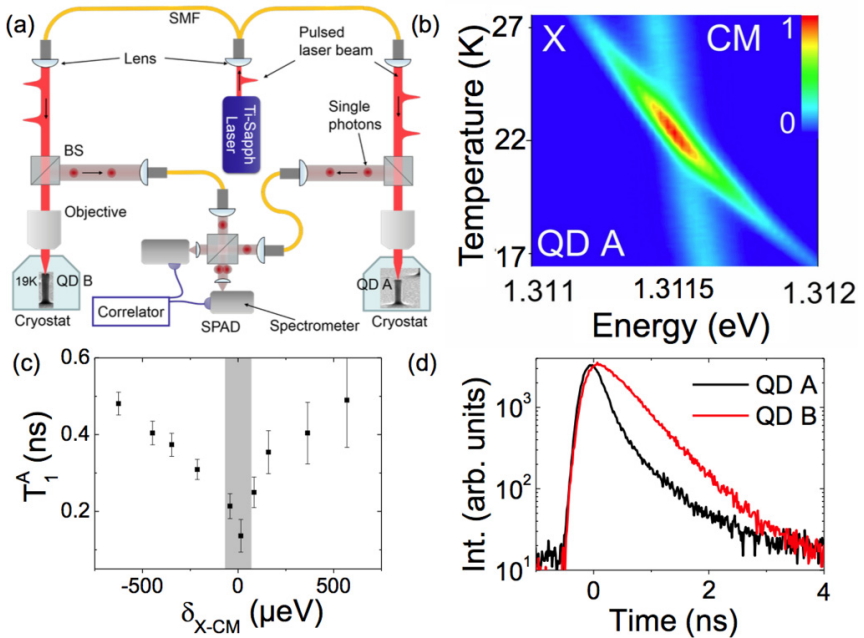

(d)

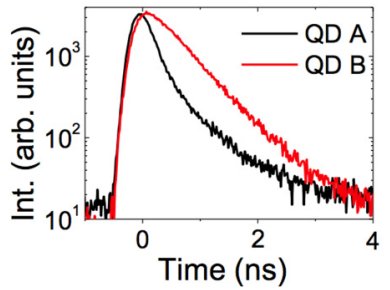

FIG. 1. (Color online) (a) Scheme of the experimental setup (see text for details). (b) QD A emission as a function of temperature and energy. The QD A exciton line $(X)$ is resonant to the bare cavity mode $(\mathrm{CM})$ at $22 \mathrm{~K}$. (c) Decay time of the QD A exciton line $\left(T_{1}^{\mathrm{A}}\right)$ deconvoluted from the temporal resolution of the setup vs $\delta_{\mathrm{CM}-X}$. The spectral range used for the two-source interference is marked with a gray vertical stripe. (d) Decay of the neutral exciton emission of QD $\mathrm{A}(\mathrm{QD} B)$ in a black (red) color line for $\delta_{\mathrm{CM}-X}=0$.

The micropillar devices are fabricated from the same planar microcavity sample consisting of a bottom (top) Bragg mirror with 32 (18) $\lambda / 4 \mathrm{GaAs} / \mathrm{Al}_{0.95} \mathrm{Ga}_{0.05}$ As layers, surrounding an adiabatic cavity embedding a dilute InGaAs QD layer in its center (see Refs. [28,29] for details). Micropillar cavities are laterally centered on single selected QDs with a $50 \mathrm{~nm}$ accuracy using the optical in situ lithography technique [30]. The cavity diameter is adjusted for each QD so as to match the cavity and QD resonances. Two in situ lithography steps were performed on two parts of the same $1 \mathrm{~cm}^{2}$ area sample so as to fabricate a dozen micropillars on each part. After the pillar etching, the sample was cleaved in two pieces, which were inserted in two separated cryostats with independent temperature tuning [see Fig. 1(a)]. Two pillars having the same diameter in each cryostat are studied, each of them deterministically coupled to a $\mathrm{QD}$, hereafter referred to as QD A and QD B.

First, we individually characterize the performance of each single photon source. A single Ti:sapphire laser, providing 3-ps-long light pulses at a $82 \mathrm{MHz}$ repetition rate, is used to excite both devices, so as to obtain synchronized single photons from each source. To obtain good single photon purity in a strong Purcell effect regime, the QD devices are excited with a laser energy below the wetting layer resonance [31]. A common excitation state, at $1.370 \mathrm{eV}$, was found for both QDs. For both devices, the QD resonance is spectrally very close to the mode resonance at $10 \mathrm{~K}$. Fine spectral matching is obtained by increasing the temperatures that shift the exciton lines to lower energies throughout the cavity mode $(\mathrm{CM})$ resonance [Fig. 1(b)]. Figure 1(c) shows the decay time of the QD A neutral exciton line as a function of the energy detuning $\delta_{\mathrm{CM}-X}=E_{\mathrm{CM}}-E_{X}$. When reducing $\delta_{\mathrm{CM}-X}$, the exciton decay time decreases to reach a minimum value of $T_{1}^{\mathrm{A}}=140 \pm 40 \mathrm{ps}$
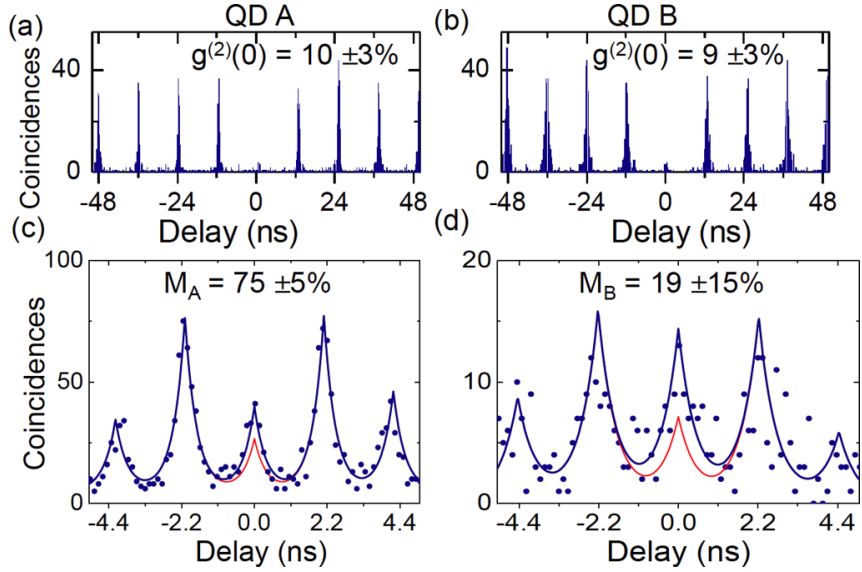

FIG. 2. (Color online) Measured autocorrelation function of (a) QD A and (b) QD B exciton lines (with 10 and 25 min acquisition time, respectively). (c), (d) Correlation histograms measuring the indistinguishability of photons successively emitted by QD A and QD B, respectively (with acquisition times of 12 and $30 \mathrm{~min}$, respectively). Black lines are fittings to the experimental data with $M_{\mathrm{A}}=75 \%$ for QD A and $M_{\mathrm{B}}=19 \%$ for QD B. As a comparison, the red lines are the calculated curves for $M=1$, considering the measured values for $g^{(2)}(0), \epsilon, R$, and $T$ values.

at resonance $\left(\delta_{\mathrm{CM}-X}=0\right)$. A similar study performed on the neutral exciton in QD B gives a lifetime of $T_{1}^{\mathrm{B}}=470 \pm 30 \mathrm{ps}$ at resonance. The difference in lifetime observed for QD A and QD B can be explained by a different coupling to acoustic phonons, resulting in a different effective Purcell effect, as recently discussed [28], or by the influence of an optically created trapped charge in the QD vicinity. The setup used for QD A could be carefully calibrated and a state-of-the-art brightness was demonstrated: At saturation, a single photon is emitted for each pulse by the QD and is collected with a $74 \pm 5 \%$ probability in the first lens. The experimental configuration on the other setup did not allow for such a precise calibration for QD B, yet we can still estimate that the source brightness is $30 \pm 10 \%$. Both sources are operated at maximum brightness for all measurements. When performing the two-source interference, the photon flux from QD A is attenuated to match that of QD B.

Single photon emission is quantified by sending the photons emitted by each device, after coupling into a single mode fiber (SMF), to a Hanbury Brown-Twiss setup: Photons impinge on a 50:50 beam splitter (BS) with outputs coupled to spectrometers for spectral filtering and single photon avalanche diodes (SPADs). Figures 2(a) and 2(b) present the autocorrelation functions measured for both devices. A good single photon purity is obtained for both with an integrated $g^{(2)}(0)$ for the zero delay peaks of $g_{\mathrm{A}}^{(2)}(0)=10 \pm 3 \%$ for QD A and $g_{\mathrm{B}}^{(2)}(0)=9 \pm 3 \%$ for QD B.

The indistinguishability of successively emitted photons for each source is measured by exciting each device twice using a 2.3 ns delay line on the excitation laser line (see the Supplemental Material [32]). The photon indistinguishability is measured through the mean wave-packet overlap $(M)$, as defined in Ref. [18]. For perfectly indistinguishable photons $M=1, g^{(2)}(0)=0$, and a perfect experimental setup, no 
signal should be observed at zero delay. Obtaining a good wave-packet overlap for successively emitted photons requires properly choosing the excitation wavelength for each source [17]. With the experimental limitation of using the same excitation line for both sources, we chose to maximize the indistinguishability on one source (QD A) that also presents the strongest effective Purcell enhancement. Under these excitation conditions, we measure $M$ for each source: It is as high as $M_{\mathrm{A}}=75 \pm 5 \%$ for QD A, but only $M_{\mathrm{B}}=19 \pm 15 \%$ for QD B [Figs. 2(c) and 2(d)].

The indistinguishability of photons emitted by a QD is mostly limited by charge noise $[17,26,33]$ that can lead to a time-dependent variation of the $X$ energy (spectral diffusion) or pure dephasing mechanisms, depending on the time scale of the charge fluctuations [34]. Here, we can equally well account for all our experimental observations (including the two-source interference presented below), with both approaches. Thus, we use a pure dephasing description which allows one to derive analytical equations for the two-source interference. In this framework, the indistinguishability of sequentially emitted photons by a single source is given by $M=\frac{T_{2}^{*}}{T_{2}^{*}+2 T_{1}}=\frac{\gamma}{\gamma+\gamma^{*}}$, where $T_{1}(\gamma)$ is the decay time (rate) of the $X$ transition and $T_{2}^{*}\left(\gamma^{*} / 2\right)$ is the pure dephasing time (rate). We deduce a dephasing $T_{2}^{*}$ between 500 and $1500 \mathrm{ps}$ (50 and $450 \mathrm{ps}$ ) for QD A $(\mathrm{QDB})$ considering $T_{1}^{\mathrm{A}}=140 \pm 40 \mathrm{ps}\left(T_{1}^{\mathrm{B}}=500 \pm 30 \mathrm{ps}\right)$.

We now characterize the two-source interference by measuring the photon coalescence for different spectral detunings of the sources: QD B is kept to a fixed temperature where $\delta_{\mathrm{CM}-X}^{\mathrm{B}}=0$ and the temperature of QD A is varied over $1.7 \mathrm{~K}$ to tune the spectral detuning $\Delta E=E_{X}^{\mathrm{B}}-E_{X}^{\mathrm{A}}$ of the two sources [Fig. 3(a)]. The QD excitations are respectively delayed so that the generated single photons impinge simultaneously on the two inputs of the BS. The photon correlation on the two output SPADs allows deducing the mean wave-packet overlap for the two sources $M_{(\mathrm{A}+\mathrm{B})}$ using the area $A_{0}$ of the zero delay peak (see the Supplemental Material). Figure 3(b) presents the zero delay peak of the correlation curve for the sources detuning $\Delta E=-3 \mu \mathrm{eV}$ (black) and $+85 \mu \mathrm{eV}$ (red). A clear decrease of the zero delay peak area is observed when the two sources are in resonance, showing an increased photon coalescence probability. Figure 3(c) presents the measured $M_{(\mathrm{A}+\mathrm{B})}$ as a function of $\Delta E$. For large detunings, it is observed that $M_{(\mathrm{A}+\mathrm{B})}=0$, as expected for distinguishable photons. At $\Delta E=0$, a mean wave-packet overlap $M_{(\mathrm{A}+\mathrm{B})}=40 \pm 4 \%$ is observed, a value largely exceeding $M_{\mathrm{B}}$. Moreover, the twophoton interference takes place on a spectrally broad spectral range, with a full width at half maximum of $\sim 15 \pm 5 \mu \mathrm{eV}$, three times larger than previous observations with QDs in a planar structure [25]. In the following, we discuss how the two-source interference is enhanced by use of the Purcell effect.

If both sources undergo pure dephasing, $M_{(\mathrm{A}+\mathrm{B})}$ for the two-photon overlap is

$$
M_{(\mathrm{A}+\mathrm{B})}=\frac{\gamma_{\mathrm{A}} \gamma_{\mathrm{B}}}{\gamma_{\mathrm{A}}+\gamma_{\mathrm{B}}} \frac{\gamma_{\mathrm{A}}+\gamma_{\mathrm{B}}+\gamma_{\mathrm{A}}^{*}+\gamma_{\mathrm{B}}^{*}}{\left(\frac{\Delta E}{\hbar}\right)^{2}+\frac{1}{4}\left(\gamma_{\mathrm{A}}+\gamma_{\mathrm{B}}+\gamma_{\mathrm{A}}^{*}+\gamma_{\mathrm{B}}^{*}\right)^{2}}
$$

(see the Supplemental Material).
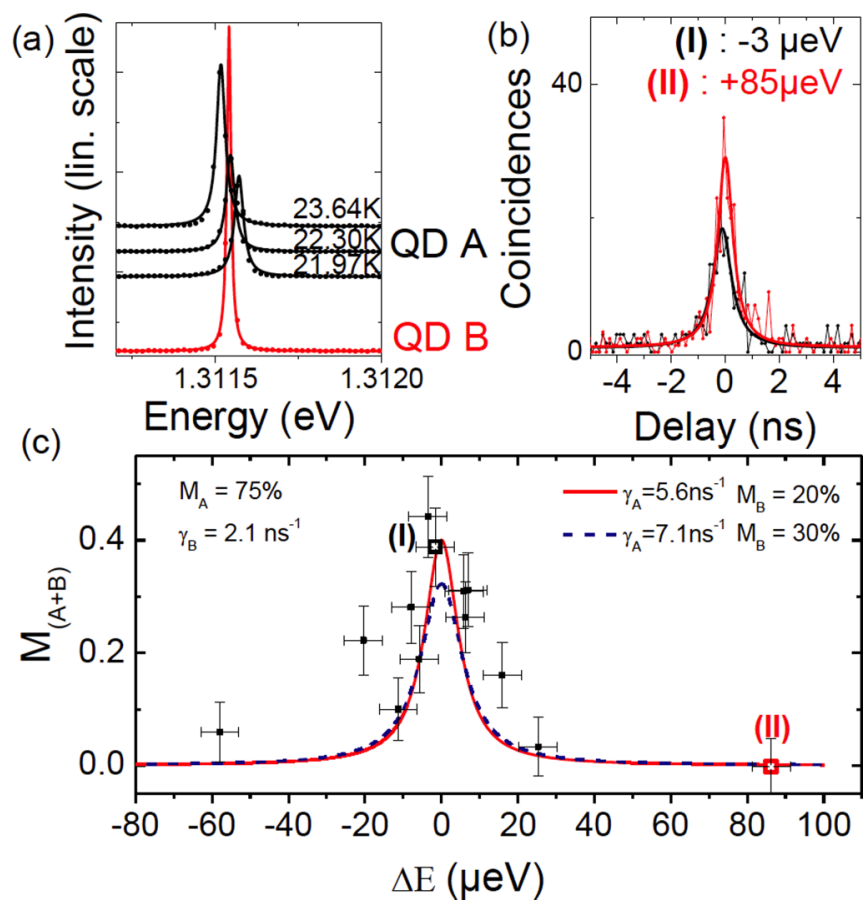

FIG. 3. (Color online) (a) Emission spectra for QD B at $19 \mathrm{~K}$ (bottom red line) and QD A for various temperatures (black lines). (b) Measured correlation of the two-source interference at two values of $\Delta E$ : (I) $-3 \mu \mathrm{eV}$ (black) and (II) $+85 \mu \mathrm{eV}$ (red). Solid lines are fits to the experimental data. (c) Measured $M_{(\mathrm{A}+\mathrm{B})}$ as a function of $\Delta E$. Lines are fits to the experimental data using the parameters indicated in the legend. The particular detunings (I) and (II) are labeled in the panel. The acquisition times of the histograms shown in (b) and the experimental points in (c) were $\sim 13$ min on average.

The linewidth of the two-photon interference is thus given by the sum of each emitter linewidth. The strong Purcell effect on QD A, contributing through $\gamma_{\mathrm{A}}$, leads to a significant increase of the spectral range over which the two-source quantum interference takes place.

As shown in Fig. 3(c), our experimental observations can be well reproduced with $M_{\mathrm{A}}=0.75, \gamma_{\mathrm{B}}=2.1 \mathrm{~ns}^{-1}, 5.6<\gamma_{\mathrm{A}}<$ $7.1 \mathrm{~ns}^{-1}$, and $0.2<M_{\mathrm{B}}<0.3$, which are consistent with the measurements performed on each source within the error bars. The two-source coalescence probability reaches $M_{\mathrm{A}+\mathrm{B}}=$ $40 \pm 4 \%$, an intermediate value between $M_{\mathrm{A}}=75 \pm 5 \%$ and $M_{\mathrm{B}}=19 \pm 15 \%$. Yet, as discussed now, this value does not result from a simple averaging of the values obtained for each source. Controlling the spontaneous emission on QD A actually significantly improves $M_{\mathrm{A}+\mathrm{B}}$.

Figure 4(a) presents the calculated $M_{(\mathrm{A}+\mathrm{B})}$ for $\Delta E=0$ as a function of the decay time of $\mathrm{QD} A$ for various values of $M_{\mathrm{B}}$. The lifetime of $\mathrm{QD} \mathrm{B}$ is fixed to $T_{1}^{\mathrm{B}}=$ $450 \mathrm{ps}$ and $M_{\mathrm{A}}=75 \%$. The vertical dashed line indicates the situation where the two sources present the same decay time. The coalescence probability is optimal for sources with an identical lifetime only for $M_{\mathrm{A}} \approx M_{\mathrm{B}}$. When $M_{\mathrm{B}}<M_{\mathrm{A}}$, the maximum probability of coalescence is obtained for an optimal value of $T_{1}^{\mathrm{A}}\left(T_{1, \mathrm{opt}}^{\mathrm{A}}\right)$ that depends on $M_{\mathrm{B}}$ : The lower the 

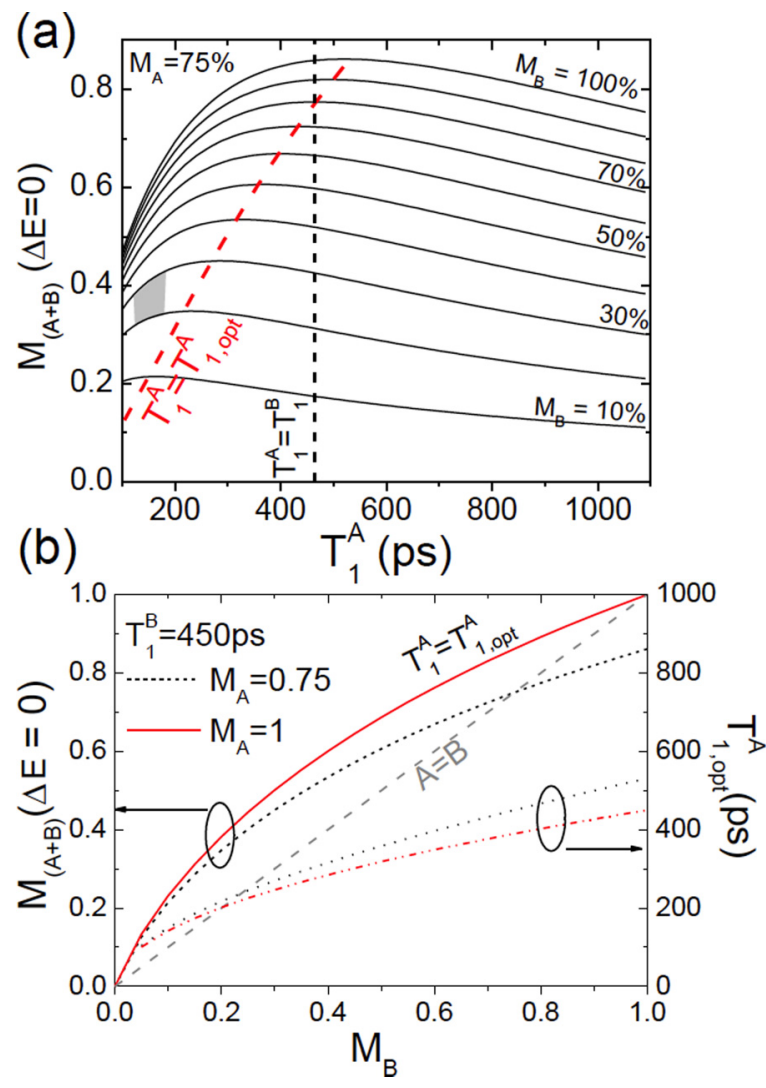

FIG. 4. (Color online) (a) Calculated photon overlap $M_{(\mathrm{A}+\mathrm{B})}$ at $\Delta E=0$ as a function of QD A decay time $T_{1}^{\mathrm{A}}$ (with a fixed $M_{\mathrm{A}}=$ $75 \%$ ) for different values of $M_{\mathrm{B}}$. The decay time of QD B is fixed at $T_{1}^{\mathrm{B}}=470 \mathrm{ps}$. The red dashed line indicates the optimal values of $T_{1, \mathrm{opt}}^{\mathrm{A}}$ where $M_{(\mathrm{A}+\mathrm{B})}(\Delta E=0)$ is maximum. The vertical dashed line indicates the situation where the two sources present the same decay time. (b) Left vertical axis: Optimal photon overlap $M_{(\mathrm{A}+\mathrm{B})}(\Delta E=0)$ obtained at $T_{1, \mathrm{~A}}=T_{1, \mathrm{opt}}^{\mathrm{A}}$ and $T_{1}^{\mathrm{B}}=470 \mathrm{ps}$ shown in a solid red (dashed black) line for $M_{\mathrm{A}}=1\left(M_{\mathrm{A}}=0.75\right)$. The gray dashed line indicates the indistinguishability when both sources are identical. Right vertical axis: $T_{1, \mathrm{opt}}^{\mathrm{A}}$ decay time as a function of $M_{\mathrm{B}}$ for a value of $M_{\mathrm{A}}=1(0.75)$ shown in a red dotted-dashed (black dotted) line. indistinguishability of successively emitted photons by the QD B source, the stronger the Purcell factor on QD A should be to overcome the pure dephasing on QD B. In other words, the faster are the decoherent processes on $\mathrm{QD} \mathrm{B}$, the faster QD A emission should be to compensate them. The gray area in Fig. 4(a) presents the conditions reached in the current measurements where this effect is demonstrated.

For the optimal value $T_{1, \mathrm{opt}}^{\mathrm{A}}$, the coalescence probability of the two-source interference significantly exceeds $M_{\mathrm{B}}$. Figure 4(b) presents in the left vertical axis the best achievable overlap $M_{(\mathrm{A}+\mathrm{B})}$ as a function of $M_{\mathrm{B}}$ obtained with $T_{1}^{\mathrm{A}}=T_{1, \mathrm{opt}}^{\mathrm{A}}$ for the case of the present measurement $M_{\mathrm{A}}=75 \%$ (black dotted line) and for $M_{\mathrm{A}}=100 \%$ (red solid line). The gray dashed line allows one to compare the best achievable overlap to the case where both sources are identical. The right vertical axis shows the value of $T_{1, \mathrm{opt}}^{\mathrm{A}}$ as a function of $M_{\mathrm{B}}$ that renders the optimal coalescence probability $M_{(\mathrm{A}+\mathrm{B})}$. For $M_{\mathrm{B}}<M_{\mathrm{A}}$, the best achievable overlap in the two-source experiment is significantly larger than the one obtained for photons successively emitted by QD B and exceeds the one obtained considering a source with the same lifetime.

In solid-state systems, where dephasing is a limitation to scalability, the two-photon interference from distinct sources is often maximized through strong temporal filtering [35], resulting in a strong reduction of the photon rate. Here, we show that cavity quantum electrodynamics, known to greatly improve the photon rate, is also a powerful asset in two-source experiments. The Purcell effect can also be used to enhance the quantum interference of remote sources. This result is crucial for the scalability of QD-based quantum networks, where the imperfections of one device can be efficiently compensated by a highly indistinguishable single photon source with a controlled lifetime.

This work was partially supported by the ERC Starting Grant No. 277885 QD-CQED, the French RENATECH network, the Labex NanoSaclay, the CHISTERA project SSQN, and the EU FP7 Grant No. 618078 (WASPS). C.A. acknowledges financial support from the Spanish FPU scholarship.
[1] M. Bayer, O. Stern, P. Hawrylak, S. Fafard, and A. Forchel, Nature (London) 405, 923 (2000).

[2] P. Michler, A. Kiraz, C. Becher, W. V. Schoenfeld, P. M. Petroff, L. Zhang, E. Hu, and A. Imamoğlu, Science 290, 2282 (2000).

[3] N. Akopian, N. H. Lindner, E. Poem, Y. Berlatzky, J. Avron, D. Gershoni, B. D. Gerardot, and P. M. Petroff, Phys. Rev. Lett. 96, 130501 (2006).

[4] R. M. Stevenson, R. J. Young, P. Atkinson, K. Cooper, D. A. Ritchie, and A. J. Shields, Nature (London) 439, 179 (2006).

[5] C. Emary, X. Xu, D. G. Steel, S. Saikin, and L. J. Sham, Phys. Rev. Lett. 98, 047401 (2007).

[6] D. Press, T. D. Ladd, B. Zhang, and Y. Yamamoto, Nature (London) 456, 218 (2008).

[7] B. D. Gerardot, D. Brunner, P. A. Dalgarno, P. Ohberg, S. Seidl, M. Kroner, K. Karrai, N. G. Stoltz, P. M. Petroff, and R. J. Warburton, Nature (London) 451, 441 (2008).
[8] M. A. Pooley, D. J. P. Ellis, R. B. Patel, A. J. Bennett, K. H. A. Chan, I. Farrer, D. A. Ritchie, and A. J. Shields, Appl. Phys. Lett. 100, 211103 (2012).

[9] Y.-M. He, Y. He, Y.-J. Wei, D. Wu, M. Atatüre, C. Schneider, S. Höfling, M. Kamp, C.-Y. Lu, and J.-W. Pan, Nat. Nanotechnol. 8, 213 (2013).

[10] O. Gazzano, M. P. Almeida, A. K. Nowak, S. L. Portalupi, A. Lemaître, I. Sagnes, A. G. White, and P. Senellart, Phys. Rev. Lett. 110, 250501 (2013).

[11] J. Claudon, J. Bleuse, N. S. Malik, M. Bazin, P. Jaffrennou, N. Gregersen, C. Sauvan, P. Lalanne, and J.-M. Gérard, Nat. Photonics 4, 174 (2010).

[12] A. Laucht, S. Pütz, T. Günthner, N. Hauke, R. Saive, S. Frédérick, M. Bichler, M.-C. Amann, A. W. Holleitner, M. Kaniber, and J. J. Finley, Phys. Rev. X 2, 011014 (2012).

[13] M. E. Reimer, G. Bulgarini, N. Akopian, M. Hocevar, M. B. Bavinck, M. A. Verheijen, E. P. A. M. Bakkers, 
L. P. Kouwenhoven, and V. Zwiller, Nat. Commun. 3, 737 (2012)

[14] M. Munsch, N. S. Malik, E. Dupuy, A. Delga, J. Bleuse, J.-M. Gérard, J. Claudon, N. Gregersen, and J. Mørk, Phys. Rev. Lett. 110, 177402 (2013).

[15] K. H. Madsen, S. Ates, J. Liu, A. Javadi, S. M. Albrecht, I. Yeo, S. Stobbe, and P. Lodahl, Phys. Rev. B 90, 155303 (2014).

[16] M. Davanço, M. T. Rakher, W. Wegscheider, D. Schuh, A. Badolato, and K. Srinivasan, Appl. Phys. Lett. 99, 121101 (2011).

[17] O. Gazzano, S. Michaelis de Vasconcellos, C. Arnold, A. Nowak, E. Galopin, I. Sagnes, L. Lanco, a. Lemaître, and P. Senellart, Nat. Commun. 4, 1425 (2013).

[18] C. Santori, D. Fattal, J. Vuckovic, G. S. Solomon, and Y. Yamamoto, Nature (London) 419, 594 (2002).

[19] C. Matthiesen, A. N. Vamivakas, and M. Atatüre, Phys. Rev. Lett. 108, 093602 (2012).

[20] R. Proux, M. Maragkou, E. Baudin, C. Voisin, P. Roussignol, and C. Diederichs, Phys. Rev. Lett. 114, 067401 (2015).

[21] Y.-J. Wei, Y.-M. He, M.-C. Chen, Y.-N. Hu, Y. He, D. Wu, C. Schneider, M. Kamp, S. Höfling, C.-Y. Lu, and J.-W. Pan, Nano Lett. 14, 6515 (2014).

[22] M. Müller, S. Bounouar, K. D. Jöns, M. Glässl, and P. Michler, Nat. Photonics 8, 224 (2014).

[23] S. Varoutsis, S. Laurent, P. Kramper, A. Lemaître, I. Sagnes, I. Robert-Philip, and I. Abram, Phys. Rev. B 72, 041303 (2005).

[24] E. B. Flagg, A. Muller, S. V. Polyakov, A. Ling, A. Migdall, and G. S. Solomon, Phys. Rev. Lett. 104, 137401 (2010).
[25] R. B. Patel, A. J. Bennett, I. Farrer, C. A. Nicoll, D. A. Ritchie, and A. J. Shields, Nat. Photonics 4, 632 (2010).

[26] P. Gold, A. Thoma, S. Maier, S. Reitzenstein, C. Schneider, S. Höfling, and M. Kamp, Phys. Rev. B 89, 035313 (2014).

[27] W. B. Gao, P. Fallahi, E. Togan, A. Delteil, Y. S. Chin, J. MiguelSanchez, and A. Imamoğlu, Nat. Commun. 4, 2744 (2013).

[28] S. L. Portalupi, G. Hornecker, V. Giesz, T. Grange, A. Lemaître, J. Demory, I. Sagnes, N. D. Lanzillotti-Kimura, L. Lanco, A. Auffèves, and P. Senellart, Nano Lett. (2015).

[29] M. Lermer, N. Gregersen, F. Dunzer, S. Reitzenstein, S. Höfling, J. Mørk, L. Worschech, M. Kamp, and A. Forchel, Phys. Rev. Lett. 108, 057402 (2012).

[30] A. Dousse, L. Lanco, J. J. Suffczyński, E. Semenova, A. Miard, A. Lemaître, I. Sagnes, C. Roblin, J. Bloch, and P. Senellart, Phys. Rev. Lett. 101, 267404 (2008).

[31] V. Giesz, O. Gazzano, A. K. Nowak, S. L. Portalupi, A. Lemaître, I. Sagnes, L. Lanco, and P. Senellart, Appl. Phys. Lett. 103, 033113 (2013).

[32] See Supplemental Material at http://link.aps.org/supplemental/ 10.1103/PhysRevB.92.161302 for details on the experimental method and on the theory on the two source wavepacket.

[33] A. V. Kuhlmann, J. Houel, A. Ludwig, L. Greuter, D. Reuter, A. D. Wieck, M. Poggio, and R. J. Warburton, Nat. Phys. 9, 570 (2013).

[34] A. Berthelot, I. Favero, G. Cassabois, C. Voisin, C. Delalande, P. Roussignol, R. Ferreira, and J. M. Gérard, Nat. Phys. 2, 759 (2006).

[35] H. Bernien, L. Childress, L. Robledo, M. Markham, D. Twitchen, and R. Hanson, Phys. Rev. Lett. 108, 043604 (2012). 